5 Marshall FF, Dietrick DD, Baumgartner WA, Reitz BA. Surgical management of renal cell carcinoma with intracaval neoplastic extension above the hepatic veins. J Urol 1988;139:1166-72

6 Moinzadeh A, Libertino JA. Prognostic significance of tumor thrombus level in patients with renal cell carcinoma and venous tumor thrombus extension. Is all T3b the same? J Urol 2004;171:598-601

\section{Streptococcus mutans endocarditis: beware of the 'diphtheroid'}

\author{
S Schelenz MD MRCPath ${ }^{1}$ A J F Page FRCP² \\ A M Emmerson FRCPath FMedSci ${ }^{3}$
}

J R Soc Med 2005;98:420-421

Streptococcus mutans, a major cause of dental caries and endocarditis, is easily dismissed by the inexperienced as a common non-pathogenic skin bacterial contaminant termed 'diphtheroid'.

\section{CASE HISTORY}

A man of 62 was admitted after three months of lethargy, malaise, night sweats and weight loss. Type 2 diabetes mellitus had been diagnosed at about the time these symptoms began and, on a home visit, his general practitioner had thought they were due to poor diabetic control. The patient had not experienced chest pain, cough, rigors or arthralgia. His medical history included a Ross procedure (pulmonary valve autograft to aortic position, pulmonary valve homograft) at age 35 for a congenital abnormality of the aortic valve. His medication was gliclazide and aspirin.

On examination he was pyrexial $\left(38.2^{\circ} \mathrm{C}\right)$, and tachycardic. The principal abnormal physical sign was a mid-systolic murmur. There was no rash or splenomegaly. Haemoglobin was $11.6 \mathrm{~g} / \mathrm{L}$, white cell count $21.6 \times 10^{9} / \mathrm{L}$ (neutrophils 95\%), C-reactive protein $72 \mathrm{mg} / \mathrm{L}$, erythrocyte sedimentation rate $94 \mathrm{~mm} / \mathrm{L}$, random blood glucose $13.1 \mathrm{mmol} / \mathrm{L}$, HbA1c 9.6\%. The initial diagnosis was hyperosmolar non-ketotic hyperglycaemia. Because of his persistent pyrexia, a set of peripheral blood cultures was taken which became positive within $24 \mathrm{~h}$ showing Grampositive rods. Since a biochemical test for the identification

Departments of ${ }^{1}$ Microbiology and ${ }^{2}$ Cardiology, Norfolk and Norwich University Hospital, Norwich NR2 3TX; ${ }^{3}$ Department of Microbiology, Queen's Medical Centre, Nottingham NG7 2UH, UK

Correspondence to: Dr Silke Schelenz

E-mail: silke.schelenz@nnuh.nhs.uk of Gram-positive rods gave no profile, the isolate was reported as 'diphtheroids' and presumed to be a contaminant. However, because of his previous valve replacement and his ongoing fevers the clinicians were advised to take more blood cultures. Three further sets of blood cultures became positive within two days. Grampositive rods were again seen; and, on review of the original Gram films, the organisms were deemed more likely to be streptococci, possibly $S$. mutans. It was strongly suggested to exclude endocarditis in this patient and to review the state of his teeth. A transthoracic echocardiogram showed a small mobile vegetation on a thickened pulmonary valve. He was then treated for presumed streptococcal endocarditis with gentamicin $80 \mathrm{mg}$ twice daily for two weeks and vancomycin twice daily for five weeks (he was allergic to penicillin). Eight teeth with advanced caries were removed while he was in hospital. His infection markers became normal.

Originally, the laboratory performed a biochemical test (API) for the identification of streptococci, which named the organisms as Leuconostoc sp. This was unlikely to be correct since the isolate was vancomycin sensitive whereas Leuconostoc spp. are resistant. After two weeks, the reference laboratory confirmed the isolate to be a fully sensitive $S$. mutans.

\section{COMMENT}

S. mutans belongs to a group of non-haemolytic Grampositive cocci which typically appear rod-shaped on acid culture medium but show a streptococcal appearance, in chains, when subcultured into a neutral or alkaline broth. The organism was first described by Clarke in 1924, who noted the variation of morphology with the $\mathrm{pH}$ of the medium. ${ }^{1}$ On the basis of their rod-shaped appearance when isolated from blood culture medium they can be easily dismissed as diphtheroids - i.e. non-pathogenic skin bacteria. In 1977 Emmerson and Eykyn² drew attention to this hazard, by reporting two cases of $S$. mutans endocarditis in which the organisms had been initially misidentified as diphtheroid contaminants.

Although diphtheroids are the second most common bacterial contaminants in blood cultures, the case presented here illustrates that 27 years on we still fall into the same trap of misidentifying an important pathogen and potentially delaying the diagnosis and treatment of a serious infection. ${ }^{3}$ Clinicians and microbiology staff need to be critical about the identification of 'diphtheroids' from blood cultures. New technology such as commercial identification kits may even add to the confusion.

\section{REFERENCES}

1 Clarke JK. On the bacterial factor in the aetiology of dental caries. $\mathrm{Br} J$ Exper Pathol 1924;5:141-7 
2 Emmerson AM, Eykyn S. Streptococcus mutans endocarditis - a trap for the unwary. BMJ 1977;i:905

3 Richter SS, Beekmann SE, Diekema DJ et al. Minimizing the workup of blood culture contaminants: implementation and evaluation of a laboratory-based algorithm. J Clin Microbiol 2002;40:2437-44

\section{Intravitreal triamcinolone acetonide for refractory diabetic macular oedema}

\author{
Arosha I Fernando BSc MRCOphth \\ Jyotin C Pandit MSc FRCSEd
}

J R Soc Med 2005;98:421-422

Diabetic macular oedema is caused by hyperpermeability of the retinal vasculature and results in retinal thickening due to intraretinal fluid accumulation between the inner and outer plexiform layers. The Early Treatment Diabetic Retinopathy Study defined clinically significant macular oedema (CSMO) by the presence of one or more of the following: retinal thickening at or within $500 \mu \mathrm{m}$ of the centre of the macula; hard exudates at or within $500 \mu \mathrm{m}$ of the macular centre, if associated with adjacent retinal thickening; retinal thickening greater than or equal to one disc area, any part of which is within one disc diameter of the centre of the macular centre. ${ }^{1}$ Focal argon laser photocoagulation is beneficial in non-ischaemic CSMO but less so in diffuse macular oedema. ${ }^{2} \mathrm{~A}$ treatment now used for macular oedema in various diseases is intravitreal triamcinolone acetonide.

\section{CASE HISTORY}

A man of 46 sought advice after two days of bilateral photophobia with red, watery eyes. 8 years before, noninsulin-dependent diabetes had been diagnosed, but he was otherwise well. On examination, unaided visual acuity was $6 / 18$ (improving to $6 / 6$ pinhole) in the right eye and $6 / 6$ in the left. He was diagnosed as having right-sided nongranulomatous uveitis without systemic associations. There were no signs of diabetic retinopathy or cystoid macular oedema. After two weeks of topical steroid treatment the uveitis resolved completely with visual improvement to $6 / 6$ unaided bilaterally. Three months later, right diabetic maculopathy developed. CSMO was absent, so focal laser treatment was not indicated at this stage. Six months later, visual acuity had declined from $6 / 6$ to $6 / 12$ in his right eye

Western Eye Hospital, 171 Marylebone Road, London NW1, UK

Correspondence to: Miss A I Fernando

E-mail: aroshaf@aol.com and CSMO was now evident, with macular thickening on ocular coherence tomography. Fluorescein angiography was performed before focal macular argon laser treatment. Three months post-laser there was little angiographic change, and because of the cystoid nature of his macular oedema it was felt that, if his visual acuity decreased, intravitreal triamcinolone should be tried. CSMO then developed in the left eye, and fluorescein angiography indicated that this was suitable for focal laser treatment; however, the right eye showed ischaemic maculopathy (Figure 1) for which laser treatment was thought inappropriate. A month later the patient's unaided visual acuity was $6 / 24$ in the right eye and 6/18 in the left eye, without improvement with a pinhole. He refused laser treatment for the left eye but agreed to intravitreal triamcinolone on the right. $4 \mathrm{mg}$ triamcinolone was injected into the right globe under sterile conditions. After a month visual acuity in this eye had improved to 6/9. On ocular coherence tomography, retinal thickness had lessened from $527 \mu \mathrm{m}$ to $168 \mu \mathrm{m}$ (Figure 2). This improvement was still evident six months post-treatment.

\section{COMMENT}

Focal macular oedema is characterized by leakage from microaneurysms and dilated capillary segments, whereas in diffuse macular oedema the leakage results from a generalized breakdown of the blood-retinal barrier, with formation of large cystoid spaces. The disappointing results with focal laser photocoagulation in conditions such as ischaemic diabetic maculopathy have prompted evaluation of other approaches. Corticosteroids have been used orally, topically and by periocular injection in the treatment of cystoid macular oedema because of their ability to inhibit the arachidonic acid pathway and

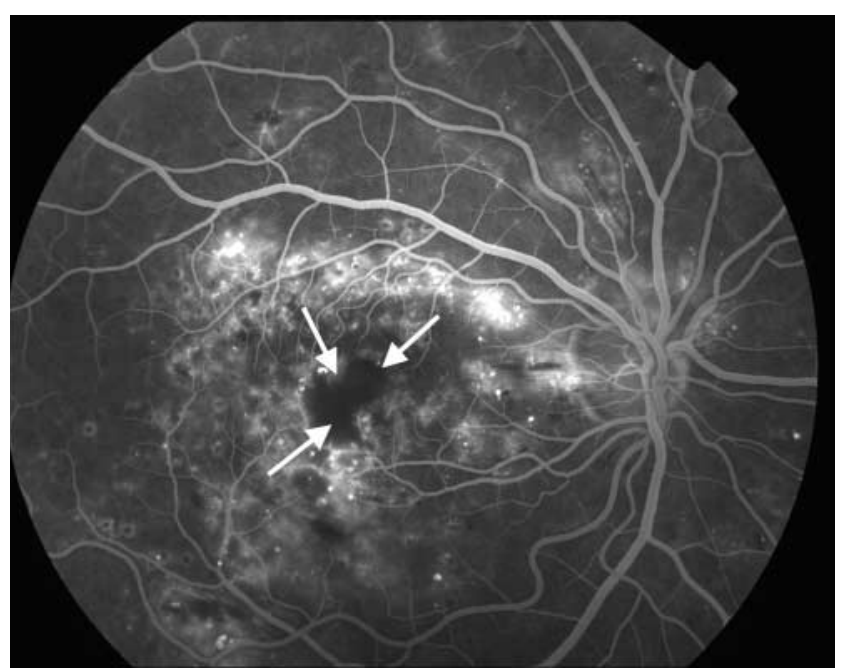

Figure 1 Fluorescein angiogram showing increased size and irregularity (arrows) of the foveal avascular zone 